\title{
Results on fractional parts of linear functions of $n$ and applications to Beatty sequences
}

\author{
TAKaO Komatsu
}

A Beatty sequence is a sequence of the form $\lfloor n \theta+\phi\rfloor$ for fixed real numbers $\theta$ and $\phi$. In order to determine the sequence $\lfloor n \theta+\phi\rfloor(n=1,2,3, \ldots)$, it is natural to consider the characteristic (or characteristic sequence) $d_{n}=\lfloor(n+1) \theta+\phi\rfloor-\lfloor n \theta+$ $\phi\rfloor(n=1,2,3, \ldots)$, from which the original sequence $\lfloor n \theta+\phi\rfloor$ can be constructed by additions only: $\lfloor n \theta+\phi\rfloor=\sum_{1=1}^{n-1} d_{i}+\lfloor\theta+\phi\rfloor(n=3,4,5, \ldots)$. Thus, the characteristic sequence determines the original Beatty sequence up to translation, and when $0<\theta<1$ it consists of 0's and 1's only.

Determining the characteristic sequence will be the main theme of this thesis, and our interest lies chiefly in the inhomogeneous case. There are many methods for deriving the homogeneous characteristic sequence, but it is known to be difficult to compute the general inhomogeneous case.

Following the introduction in Chapter I, we discuss homogeneous characteristic sequences in Chapter II, where the methods are well-known but are relevant to later chapters. The original work begins from Chapter III.

In Chapter III we consider the characteristic sequences of $\lfloor n \theta+\phi\rfloor$ by the method of Markov and Venkov [7], which uses continued fraction expansions.

In Chapter IV we discuss the fractional part of $n \theta+\phi$ and introduce the work of van Ravenstein [6]. Using the Three Gap Theorem he determined the second smallest value of $\|n \theta\|$ with $0<n<q_{i}$. We shall generalise this to the inhomogeneous case.

The sorting problem in the inhomogeneous case is also solved here. For any irrational $\theta$ we can determine the ordering $u_{1}, u_{2}, \ldots, u_{N}$ of $\{0,1,2, \ldots, N-1\}$ with $\left\{u_{0} \theta+\phi\right\}<\left\{u_{1} \theta+\phi\right\}<\cdots<\left\{u_{N-1} \theta+\phi\right\}$.

We also give an easily computable way of obtaining long sections of the inhomogeneous characteristic sequence $\lfloor(n+1) \theta+\phi\rfloor-\lfloor n \theta+\phi\rfloor$ from the homogeneous one $\lfloor(n+1) \theta\rfloor-\lfloor n \theta\rfloor$. We give several examples. To get the homogeneous characteristic

Received 13th April, 1995.

Thesis submitted to Macquarie University, December 1994. Degree approved, April 1995. Chief supervisor: Professor Alfred J. van der Poorten; Joint supervisor: Dr Peter A.B. Pleasants; Associate supervisor: Professor John H. Loxton.

Copyright Clearance Centre, Inc. Serial-fee code: 0004-9729/95 SA2.00+0.00. 
sequence we use the method of Fraenkel, Mushkin and Tassa [2], but once this is obtained, arbitrarily long sections of the original inhomogeneous characteristic sequence can quickly be derived by a series of shifts closely related to the fractional parts $\{n \theta+\phi\}$.

In Chapter $\mathrm{V}$ we give a corrected version of [5]. Their results for the homogeneous case still stand, but their results for the inhomogeneous case have needed thorough going revision which has made the proofs much longer. Their results on irrationality measure and algebraic independence are also corrected.

Shortly after [5], a similar paper by the Borwein brothers [1] appeared. Both of these papers give expressions for $\sum_{k=1}^{\infty} \sum_{1 \leqslant m \leqslant k \theta+\phi} x^{k} y^{m}$. Replacing the expression in [5] by our corrected version in Chapter $\mathrm{V}$, we can now show that the two different expressions given are equivalent. The starting point for this topic is relative continued fraction expansions. This is the content of Chapter VI.

In Chapters VII and VIII we deal with substitution invariant Beatty sequences. If we assume $0<\theta<1$, the sequence $f_{1} f_{2} f_{3} \ldots$ consists of 0 's and 1's only. Denote by $W_{0}$ and $W_{1}$ finite strings in the letter 0 and 1 . Then the sequence $\left(f_{n}\right)$ is said to be invariant under the subtitution $W$ given by $W: 0 \longrightarrow W_{0}, 1 \longrightarrow W_{1}$, if the infinite strings $f_{0}=f_{1} f_{2} f_{3} \cdots$ and $W\left(f_{\theta}\right)=W_{f_{1}} W_{f_{2}} W_{f_{3}} \cdots$ coincide. The homogeneous case $\phi=0$ and the inhomogeneous case $\phi \neq 0$ are considered in Chapter VII and Chapter VIII, respectively. Several mathematicians have already discussed this problem, but our argument is more mechanical, efficient and rather different.

Although it is also about Beatty sequences, Chapter IX is not directly related to the other chapters. Here, we investigate the sum of a fixed number of consecutive terms of a Beatty sequence. For a fixed number $h$, we give an accurate least upper bound and greatest lower bound of $\sum_{i=1}^{h}\lfloor(N+i) \theta\rfloor$ for a variable non-negative integer $N$.

\section{REFERENCES}

[1] J.M. Borwein and P.B. Borwein, 'On the generating functions of the integer part: $[n \alpha+\gamma]$ ', J. Number Theory 43 (1993), 293-318.

[2] A.S. Fraenkel, M. Mushkin and U. Tassa, 'Determination of [n$\theta]$ by its sequence of differences', Canad. Math. Bull. 21 (1978), 441-446.

[3] T. Komatsu, 'On the characteristic word of the inhomogeneous Beatty sequence', Bull. Austral. Math. Soc. 51 (1995), 337-351.

[4] T. Komatsu, 'The fractional part of $n \theta+\phi$ and Beatty sequences', J. Théorie des Nombres de Bordeaux (to appear).

[5] K. Nishioka, I. Shiokawa and J. Tamura, 'Arithmetical properties of a certain power series', J. Number Theory 42 (1992), 61-87.

[6] T. van Ravenstein, 'The three gap theorem (Steinhaus conjecture)', J. Austral. Math. 
Soc. Ser. A 45 (1988), 360-370.

[7] B.A. Venkov, Elementary number theory (Wolters-Noordhoff, Groningen, 1970).

School of Mathematics, Physics, Computing and Electronics

Macquarie University

New South Wales 2109

Australia

e-mail: komatsu@mpce.mq.edu.au 